\title{
Clinical Audit: Paediatric Medical Team Attendance at Deliveries in an Outer Metropolitan Hospital in Western Australia
}

Gelaye Tadesse Nadew. ${ }^{1}$

\begin{abstract}
Background: The attendance of paediatric medical teams at deliveries has become accepted as an integral part of birth management under current multidisciplinary team arrangements. Under current guidelines, specific clinical indicators have been developed to determine whether paediatric teams need to attend deliveries. High rates of attendance indicate that paediatric medical team resources are being extended beyond the criteria at the expense of essential services being provided elsewhere. Methods: A retrospective clinical audit was used to evaluate the frequency of paediatric team attendance and related clinical indicators. Deliveries were recorded and audited for a randomly selected calendar month. Paediatric team attendance was evaluated against foetal distresses, types of delivery, gestational age and birth weight. Results: A total of 112 deliveries were recorded for the selected calendar month and paediatric medical teams attended $74.1 \%(n=80)$ of the deliveries. Participants were comprised of $50.9 \%(n=57)$ male and $49.1 \%(n=55)$ female babies. Of these deliveries, $66.7 \%(n=72)$ were term, $30.6 \%(n=33)$ post-term and $2.8 \%(n=3)$ pre-term. A total of $69.4 \%(n=75)$ of deliveries were spontaneous vaginal deliveries (SVD), while $22.2 \%(n=24)$ were caesarean sections and $8.3 \%(n=9)$ were instrumental deliveries. Foetal distress was experienced by $16.2 \%(n=17)$ of babies. Conclusion: Paediatric medical team attendance at deliveries was higher than the combined rate of caesarean sections, pre-term babies, instrumental deliveries and reported cases of foetal distress.
\end{abstract}

Keywords: Fetal Distress; Cesarean Section; Pediatrics; Clinical Audit (Source: MeSH-NLM).

About the Author: Gelaye Tadesse Nadew is a final year medical student of the 4-year MD program at oceania University of Medicine, Apia, Samoa. He also holds PhD from University of Adelaide.

\section{Introduction}

This study sought to explore the attendance of paediatric teams at deliveries in an outer suburban hospital in Western Australia. A clinical audit was undertaken using the clinical guidelines developed by the Western Australia's Women and New-born Health Service (adopted by district hospitals to guide clinical practice principles in the management of births). ${ }^{1}$ The attendance of paediatric medical teams at deliveries is enforced based on these guidelines; however, the clinical necessity of all such attendances remains unclear as the majority of deliveries do not constitute emergency or other clinical indicators as outlined in the guidelines. Further, these attendances could result in paediatric medical team resources being over used. As a result, this clinical audit was undertaken to evaluate the attendances of paediatric medical teams in relation to clinically identified needs.

Although clinical audits have widely been used to improve services, ${ }^{2}$ a literature search of PubMed, Medline and PubMed Central using key terms (e.g., paediatric team, management of birth, foetal distress and the role of paediatric teams during birth) produced few results. Thus, it appears that the attendance of paediatric medical teams at deliveries has not been adequately investigated. This may explain the lack of clarity that exists in relation to defining the role of paediatric teams in bir- th management. A systematic and comprehensive evaluation of current practice will lead to clarity of the role of paediatric, refining of clinical indicators, and prioritisation of attendances with appropriate level of skills without depleting essential services elsewhere. The pitfall of current practice was stipulated by a study in South Africa audited the attendance of paediatric teams at deliveries and found that paediatric team resources were being over used and their attendance purposes were not clearly defined..$^{3-5}$ Another study showed the importance of paediatric medical teams attending deliveries to ensure 'best outcomes' by carrying out the new-born assessment early. ${ }^{6,7}$ This requires allocation of an appropriately skilled doctor who can perform resuscitation if required and carry out a new-born examination comprehensively with objectives of identifying any anomalies and set a benchmark for subsequent examinations. For the above reasons and in circumstances where risks are identified during antenatal care or during the progression of birth, the attendance of paediatric medical teams are essential and can be prearranged. ${ }^{5,6,8}$

When risks associated with birth are identified early during antenatal care, the use of paediatric team resources can be predetermined and more cost effective. ${ }^{4.5,9}$ In anticipating the unknown, soliciting paediatric team resources can offer added safety and reassurance to mothers and the delivery team di- 
rectly involved. ${ }^{16-8,10}$ However, it should be noted that these attendances may occur at the expense of essential services being provided elsewhere. ${ }^{3,4,6,11}$ Such attendances need to be considered in relation to it being a critical time of life; many serious events with lasting sequelae can occur in the first few hours or days following birth. $4,6,9$ Events that occur during the progression of birth are important and must be effectively managed to ensure that adverse events are minimised or eliminated. ${ }^{4,5,10-12}$ This underpins the need for paediatric teams to attend deliveries to ensure the best outcomes. However, the issues of efficiency and safety need to be balanced against the best outcomes. ${ }^{13,14}$

Involving a paediatric team at delivery depends on various medical factors and potential complications or risk factors being identified either during antenatal care and/or the progression of birth, 5 including maternal medical conditions, gestational age, types of delivery, breech positions, elective or emergency caesarean sections and foetal distress. Gestational age has three categories: pre-term, term and post-term. Foetal distress includes bradycardia, tachycardia and foetal hypoxia., ${ }^{3,4,5,15}$ To minimise risks, births are managed by multidisciplinary teams as outlined in the clinical guidelines.' Under these guidelines, a member of the Department of Neonatal and Paediatrics or other designated personnel will be called to attend the birth for possible resuscitation or other interventions. The guidelines state that a paediatric medical team must be given sufficient notice to enable a staff member to attend at the labour/birth suite to check and prepare the resuscitation trolley and obtain a history of the relevant pregnancy and labour details. Neonatal and Paediatric medical teams attending births include Resident Medical officers (RMOS), registrars, senior registrars and consultants. The decision to call the paediatric team is the responsibility of the obstetric staff. The guidelines further state that the obstetrics department must contact the appropriate member of the Department of Newborn Services to discuss any possible issues. Additionally, caesarean sections must not commence unless a medical member of the Department of Neonatal Paediatrics is present in the theatre.

According to the guidelines, a paediatric RMO is required to attend any delivery involving forceps and vacuum extraction (low cavity), pre-eclampsia, intrauterine growth restriction, a membrane that ruptured more than 24 hours earlier (if no antibiotics were administered four hours prior to birth), maternal sepsis, elective caesarean sections for non-complicated term infants under regional anaesthesia, maternal diabetes (if the mother required insulin during pregnancy and/or labour), cases where maternal morphine analgesia was administered within four hours of the delivery and any cases where gestation is less than 37 weeks.'

Conversely, both a paediatric RMO and registrar are required to attend any delivery involving high- or mid- cavity forceps/ vacuum extraction, trials of instrumental births in theatres, elective caesarean sections, breech births, cases where the gestational age is less than 35 weeks or the mother has a poor obstetric history (e.g., previous perinatal and neonatal death) or multiple pregnancies and cases where gestation is less than 38 weeks and more than 41 weeks. All non-elective caesarean sections or those performed under general anaesthesia, me- conium stained amniotic fluid, foetal bradycardia, other acute foetal compromise, rhesus isoimmunisation, antepartum haemorrhage/intrapartum bleed also require the attendance of a paediatric RMO and registrar.'

Code 'Blue' paediatric births refer to more complicated conditions and require the attendance of a RMO and registrar or, in their absence, a neonatal consultant and include all births of 30 weeks gestation or less, multiple pregnancies of 34 weeks gestation or less, severe acute foetal compromise, severe rhesus isoimmunisation (e.g., hydrops) other very high risk births and known high risk congenital anomalies (e.g., diaphragmatic hernia).' These guidelines are consistent with international standards for birth management and standard of practices outlined by the Australia New Zealand College of Obstetricians and Gynaecologists and the Australian College of Physicians.,12,13

\section{Methods}

A retrospective clinical audit of paediatric medical team attendance of deliveries was undertaken in a hospital located in the outer suburbs of Western Australia. The month of September 2014 was randomly selected and the medical records for all births registered that month were obtained from the Medical Record Department for the audit.

It was hypothesised that a significant number of attendances by paediatric teams at birth would form part of routine practices and would not be clinically required. Based on this hypothesis, the study evaluated the frequency of paediatric medical team's attendances at births and the clinical reasons for these attendances.

A clinical audit of the empirical data was undertaken to compare paediatric medical team attendances against clinical indicators. Data on key variables (i.e., gender, gestational age, birth weight, Apgar score and foetal distress during birth) were obtained and evaluated against paediatric team attendance of delivery. No specific form of recording was available to confirm the reasons for paediatric team attendance. Thus, for the purpose of this study, a paediatric team was deemed to have attended for medical reasons if they attended within 30 minutes of the birth. Three groups of gestational age categories (i.e., pre-term (up to 36.9 weeks), term ( 37 to 40 weeks) and postterm ( 40.1 weeks and above)) were created for this study.' Birth weight was grouped into four categories: up to 2600 grams, 2601-3000 grams, 3001-4000 grams and 4001 grams or more. ${ }^{1}$ The frequency of paediatric medical team attendance was evaluated using SPSS. Cross-tabulation was used to examine the relationship between each group with a focus on clinical indicators and paediatric medical team attendances. Data missing due to poor documentation are not corrected and reported as missing in this study.

Steps were undertaken to ensure the confidentiality and anonymity of data. Personal identifying features such as names, dates of birth, unit registration numbers and addresses have been omitted. The Institutional Review Board and Ethics Committee of the Oceania University of Medicine granted an ethics clearance waiver for this study. 


\section{Results}

During the selected month, 112 babies were delivered comprising $50.9 \%(n=57)$ males and $49.1 \%(n=55)$ females. The majority of deliveries (i.e., $66.7 \%$ or $n=72$ ) were term, only $2.8 \%(n=3)$ were pre-term and $30.6 \%(n=33)$ were post-term. The minimum gestational age recorded was 36.1 and the maximum was 41.3 . The mean was 39.1 with a standard deviation of 1.15. SVDs accounted for $69.4 \%(n=75)$ of deliveries and caesarean section deliveries accounted for $22.2 \%(n=24)$ (see Table 1 ).

Further analysis of caesarean sections showed that $3.7 \%(n=4)$ of these were non-elective lower uterine segment Caesarean sections (NELUSCSS), $15.7 \%(n=17)$ were elective lower uterine segment Caesarean sections (ELUSCSS) and $2.8 \%(n=3)$ were emergency lower uterine segment Caesarean section (EMLUSCS). Data for four deliveries were missing. Notably, $16.2 \%(n=17)$ of babies experienced foetal distress; however, the majority of babies ( $83.8 \%$ or $n=89$ ) did not experience foetal distress during delivery (see Table 1 ).

Of the babies born, 12 male and 5 female babies experienced foetal distress; however, 45 female and 44 male babies did not experience foetal distress during delivery. In relation to gestational age, paediatric teams attended the births of all pre-term births $(2.8 \%$ or $n=3), 46.7 \%(n=50)$ of term births and $25.2 \%$ $(n=27)$ of post-term births. Accumulatively, paediatric teams attended $74.8 \%(n=80)$ of births.

Cross-tabulating the type of delivery with paediatric team attendance showed that paediatric teams attended all caesarean sections and instrumental deliveries. Further, $43.5 \%(n=47)$ of SVDs were also attended. Additionally, paediatric teams atten-

Table 1. Paediatric Team Attendance by Cestational Age, Delivery Type and Foetal Distress

\begin{tabular}{lcc}
\hline Characteristics & Number of cases, $\mathrm{n}(\%)$ & Paediatric team attendance, $\mathrm{n}(\%)$ \\
$\begin{array}{l}\text { Cestational age (weeks) } \\
\text { Up to } 36.9 \text { (pre-term) }\end{array}$ & $3(2.7)$ & $3(2.7)$ \\
$37.0-40.0$ (term) & $72(64.3)$ & $50(46.7)$ \\
40.1 and above (post-term) & $33(29.5)$ & $27(25.2)$ \\
Delivery type & $75(69.4)$ & $47(43.5)$ \\
SVD & $24(22.2)$ & $24(22.2)$ \\
C-Section & $9(8.3)$ & $9(8.3)$ \\
Instrumental & $17(16.2)$ & $17(16.2)$ \\
Foetal Distress & $89(83.8)$ & $63(60.0)$ \\
Yes & & \\
No &
\end{tabular}

Table 2. Delivery Types and Cestational Age Groups

\begin{tabular}{lcccc}
\hline \multirow{2}{*}{ Delivery type } & \multicolumn{3}{c}{ Gestational age (weeks) } & Total \\
& Up to 36.9 & $37.0-40.0$ & 40.1 and above & \\
\hline Up to 36.9 (pre-term) & $1(1.0)$ & $48(45.7)$ & $24(22.9)$ & $73(69.5)$ \\
$37.0-40.0$ (term) & $2(1.9)$ & $18(17.1)$ & $3(2.9)$ & $23(21.9)$ \\
40.1 and above (post-term) & $0(0.0)$ & $5(4.8)$ & $4(3.8)$ & $9(8.6)$ \\
Total & $3(2.9)$ & $71(67.6)$ & $31(29.5)$ & $105(100.0)$ \\
\hline
\end{tabular}

ded $60.0 \%(n=63)$ of deliveries without identified clinical indicators (see Table 1).

A comparison of gestational age and foetal distress showed that none of the pre-term babies experienced foetal distress. Conversely, $2.9 \% \quad(n=3)$ of the term babies and $13.6 \% \quad(n=14)$ of the post-term babies experienced foetal distress (see Table 1). A comparison of gestational age and mode of deliveries showed that $45.7 \%(n=48)$ of the term deliveries and $22.7 \%$ $(n=24)$ of the post-term deliveries were by SVDs. Conversely, caesarean sections accounted for $17.1 \%(n=18)$ of term deliveries (see Table 2).

\section{Discussion}

The demographic data showed that there is no significant margin between males and females born during the calendar month selected for the study. The majority of babies were born to term, but a quarter of the babies were born post-term. Additionally, the majority of deliveries were SVDs. Less than a quarter of deliveries were by caesarean section and a small group were instrumental. Foetal distress was reported in a small group of deliveries; however, nearly three-quarters of babies did not experience foetal distress. All cases of foetal distress occurred in term and post-term deliveries. Notably, more males than females experienced foetal distress. Attendances by paediatric medical teams were higher than combined cases of foetal distress, caesarean sections and instrumental deliveries.

The findings of this study were consistent with a number of other studies that found a high rate of paediatric team attendances at deliveries. $4.5,8,11,12$ Some attendances have been shown to be unwarranted and to occur despite paediatric services being needed elsewhere. ${ }^{4}$ It has been argued that a failure to identify antenatal care risks has led to an increased risk of foetal distress and thus paediatric medical teams need to attend deliveries to manage these risks. ${ }^{13-17}$ This may be the case in developing countries where antenatal care is poorly designed or the necessary resources are not available to identify risks early and decide whether paediatric teams are required. ${ }^{18-21}$ However, in the countries where antenatal care is well developed, supported by sufficient resources (both human and technological) and hospitals situate neonatal and paediatric teams and delivery suites within close proximity to one another, there is a significant scope for reviewing paediatric team attendances. $2,4,5,18$

Leaving aside emergencies, all planned deliveries can be prebooked with paediatric teams by a simple means of communication once the patient is booked for delivery. Advanced communication of clinical details would allow paediatric teams to appropriately allocate experienced members or doctors to delivery suites. ${ }^{22-2} 4$ This study showed that there is scope to review current clinical practices and resource allocation. Paediatric team attendance was higher for pre-term babies, babies with low birth weights, caesarean sections and instrumental deliveries. Thus, current arrangements for paediatric medical teams in birth management need to be reviewed. ${ }^{22,25}$ As stated above, this study had some limitations; however, its findings could provide the bases for more comprehensive studies that cover both maternal health and foetal development during antenatal care. It is possible that some clinical indicators warranting paediatric 
team attendance may not have been captured due to inadequacies in documentation and study limitations. It is important to note that the study was limited to reviewing documents detailing foetal status and clinical episodes associated with the progression of births. Antenatal clinical data, including maternal health statuses, were not evaluated. The current study showed that inadequacies in current practices could be leading to paediatric resources being over used. However, future studies should consider comprehensive data from maternal health files, antenatal care resumes and clinicians' opinions before any changes to current practices are implemented.

This study showed that paediatric medical teams attended more deliveries than clinical indicators would suggest were necessary. Overall, paediatric attendance was significantly higher for caesarean sections, pre-term babies, instrumental deliveries and reported cases of foetal distress. Despite its limitations, the findings of this study challenge the current arrangements for paediatric medical teams attendances during deliveries. Specific criteria and guidelines set by the colleges of obstetrics and gynaecology and paediatrics provide set of clinical standards; however, it appears that clinicians are overcautious and at times attend deliveries in the absence of real clinical indicators.

In the current clinical environment, arrangements and the physical layout of hospitals situate obstetrics and gynaecology and neonatal and paediatric departments within close proximity to the delivery suites. Thus, there is scope to review the guidelines without compromising standards of practice and patients' safety. The findings do not provide a comprehensive answer; however, they do support a review of the current arrangements and highlight the need for broader further studies.

\section{References}

1. King Edward Memorial Hospital. Intrapartum care: clinical guidelines relevant to obstetrics and midwife, women and newborn health service. Perth: Government of Western Australia Department of Health; 2012.

2. National Institute for Clinical Excellence. Principles for best practice in clinical audit. Oxon (UK): Radcliffe Medical Press; 2002.

3. Tooke LJ, Joolay $Y$, Horn AR, Harrison MC. Is the attendance of paediatricians at all elective caesarean sections an effective use of resources? S Afr Med J. 2011 Sep 27;101(10): 749-50.

4. Davies, P, Miles R, Harrington J, Lawrence S. Which births are high risk? Condition of neonates at birth and risk of intervention associated with type of delivery and thickness of meconium in the district general hospital setting. Internet J Pediatr Neonatol. 2002;3(1):1-6.

5. Primhak RA, Herber SM, Whincup $G$, Milner RD. Which Deliveries require paediatricians in attendance? $\mathrm{Br}$ Med J (Clin Res Ed). 1984 Jul 7;289(6436):16-8. 6. Tudjegbe So, Imaremgiaye CO, Sadoh WE. Determinants of paediatrician's presence at Caesarean section. West Afr J Med. 2012 Jan-Mar;31(1):24-7.

7. Iyengar SD, Iyengar K, Martines JC, Dashora K, Deora KK. Childbirth practices in rural Rajasthan, India: implications for neonatal health and survival. J
Perinatol. 2008 Dec;28 Suppl 2:S23-30.

8. Lawn JE, Lee AC, Kinney M, Sibley L, Carlo WA, Paul VK, et al. Two million intrapartum-related stillbirths and neonatal deaths: where, why, and what can be done? Int J Gynaecol Obstet. 2009 Oct;107 Suppl 1:S5-S18, S19. 9. Sweeney K, Spurway J, Mein B, Magotti R, Benzie R, Challis D, et al. Congenital Epulis: a clinical case presentation. Australas J Ultrasound Med. 2014 May; $17(2): 85-8$.

10. Hope P, Breslin S, Lamont L, Lucas A, Martin D, Moore I, et al. Fatal shoulder dystocia: a review of 56 cases reported to the Confidential Enquiry into Stillbirths and Deaths in Infancy. Br J Obstet Gynaecol. 1998 Dec;105(12):1256-61. 11. Belbcowe H, Cousens S, Chou D, Oestergaard M, Say L, Moller AB, et al. Born too soon: the global epidemiology of 15 million preterm births. Reprod Health. 2013;10 Suppl 1:S2.

12. The Royal Australian and New Zealand College of Obstetricians and Gynaecologists. Standards of maternity care in Australia and New Zealand. Melbourne: The Royal Australian and New Zealand College of Obstetricians and Gynaecologists; 2014.

13. Neonatal Intensive Care Unit Delivery Team Policy for High Risk Delivery. Maternal Child Health Policy and Procedure Manual; 2015

14. Araf MA, Youssef RM, Abdel Rahman AH. Risk assessment for obstetric interventions. J Egypt Public Health Assoc. 2003;78(5-6):467-95.

15. Atherton N, Parsons SJ, Mansfield P. Attendance of paediatricians at elective Caesarean sections performed under regional anaesthesia: is it warranted? J Paediatr Child Health. 2006 Jun;42(6):332-6.

16. Niermeyer S, Kattwinkel J, Van Reempts P, Nadkarni V, Phillips B, Zideman D, et al. International Guidelines for Neonatal Resuscitation: An excerpt from the Guidelines 2000 for Cardiopulmonary Resuscitation and Emergency Cardiovascular Care: International Consensus on Science. Pediatrics. 2000 Sep;106(3): E29.

17. Thomas MR, Yoxall CW, Weeks AD, Duley L. Providing newborn resuscitation at the mother's bedside: assessing the safety, usability and acceptability of a mobile trolley. BMC Pediatr. 2014 May 29;14:135.

18. Clinical Excellence Commission. Foetal monitoring: are we getting it right? Clinical Focus Report from Review of Clinical Incident Reports. Sydney: Clinical Excellence Commission; 2013.

19. Mullany LC, Khatry SK, Katz J, Stanton CK, Lee AC, Darmstadt CL, et al. Injections during labor and intrapartum-related hypoxic injury and mortality in rural southern Nepal. Int J Gynaecol Obstet. 2013 Jul;122(1):22-6.

20. Yousaf UF, Hayat $S$, Afzal N. Resuscitation of newborn in high risk deliveries. J Ayub Med Coll Abbottabad. 2015 Apr-Jun;27(2):343-5.

21. Lee AC, Cousens S, Wall SN, Niermeyer S, Darmstadt GL, Carlo WA, et al. Neonatal resuscitation and immediate newborn assessment and stimulation for the prevention of neonatal deaths: a systematic review, meta-analysis and Delphi estimation of mortality effect. BMC Public Health. 2011 Apr 13;11 Suppl 3:S12.

22. Zupan J. Perinatal mortality in developing countries. N Engl J Med. 2005 May 19;352(20):2047-8.

23. Gordon A, McKechnie EJ, Jeffery H. Pediatric presence at caesarean section: justified or not? Am J Obstet Gynecol. 2005 Sep;193(3 Pt 1):599-605. 24. Kamath BD, Todd JK, Glazner JE, Lezotte D, Lynch AM. Neonatal outcomes after elective caesarean delivery. Obset Gynaecol. 2009 Jun;113(6):1231-8.

25. Williams KP, Farquharson DF, Bebbington M, Dansereau J, Galerneau $F$, Wilson RD, et al. Screening for fetal well-being in a high-risk pregnant population comparing the nonstress test with umbilical artery Doppler velocimetry: randomized controlled clinical trial. Am J Obstet Gynecol 2003 May; 188(5):1366-71.

\section{Acknowledgments}

None.

Conflict of Interest Statement a Funding

The author has no funding, financial relationships, or conflicts of interest to disclose.

Author Contributions

Conceptualization, Data collection, Writing, Critical revision of the manuscript, Approval of the final version: GTN.

Cite as:

Nadew GT. Clinical audit: paediatric medical team attendance at deliveries in an outer metropolitan hospital in Western Australia. Int J Med Students. 2016 Sep-Dec;4(3):104-7. 